\title{
KIMA - A Holographic Telepresence Environment Based on Cymatic Principles
}

Oliver Gingrich EngD Candidate

Bournemouth University

126 Fernhead Road

London W9 3EN

United Kingdom

oliver@musion.co.uk

Alain Renaud

Bournemouth University

126 Fernhead Road

London W9 3EN

United Kingdom

arenaud@bournemouth.ac.uk

Eugenia Emets

Artist

emetsjane@gmail.com

\author{
Oliver Gingrich, Alain Renaud, Eugenia Emets
}

\begin{abstract}
A B S T R A C T
KIMA is a holographic surround-sound installation that visualizes telepresence as both a phonetic and a synaesthetic phenomenon. The performance piece is based on the physical conditions of cymatics-the study of physically visible sound wave patterns. Two environments, a quad surround and a holographic interface, build the framework of a telematic experience that illustrates communication as wave forms while focusing on the relationship between sound and matter.
\end{abstract}

\section{Project Description}

$K I M A$ is an interactive art installation with multiple objectives: On the one hand, KIMA is designed to be a live performance that challenges conceptions of on-stage telepresence. On the other hand, KIMA is an interactive installation that can be explored by individuals. KIMA focuses on the intrinsic relationship between matter and sound, of the audio/visual. KIMA offers the chance to visually and phonetically experience how sound and image mutually influence each other, generate each other, and ultimately condition each other (Figure 1).

$K I M A$ is designed to be a user-independent installation. When not performed by live artists (a performer and a vocalist), it can be experienced by audiences as an explorative installation in which two users interact remotely_creating a phonetic field alongside a holographic visualization of this soundscape. A user in space A creates sound through voice and movement to be transmitted and visualized in space B. The user in space B modifies not only the sound environment, but equally the cymatic patterns it generates. Sound is experienced as the essence of communication. On a perceptive level, KIMA presents the user with a new form of telepresent experience: Rather than focusing on photorealism in representation, KIMA looks at sound and matter as the bricks and mortar of communication.

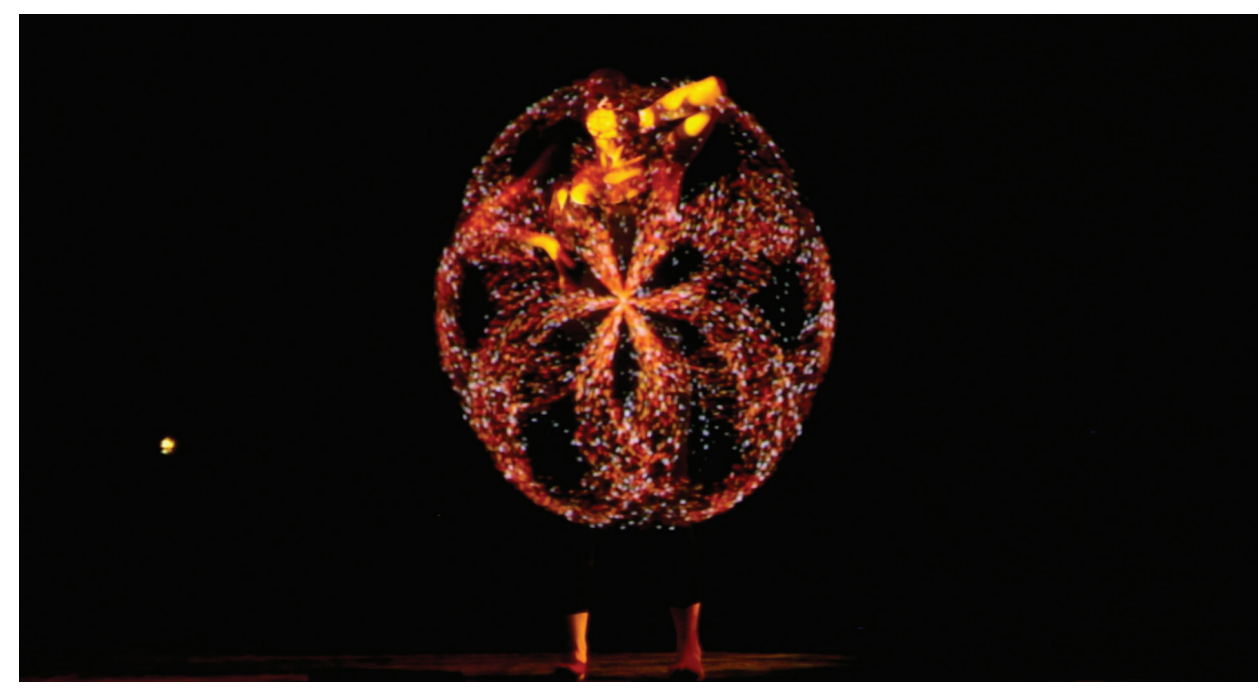

Figure 1. KIMA performed at Kinetica London. ๑) 2013 Eugenia Emets. 
KIMA is last but not least a research project, designed to investigate the relationship between sound and visuals on a very basic physical level. Cymatics is known as the area of research on the effects of vibrations, and in particular sound frequencies, on matter. Cymatics as a science can be traced to Ernst Chladni's I8th-century experiments. With a team of sound designers, a physicist, artists, and programmers, KIMA builds on these explorations as a scientific framework for sound-matter interrelations.

\section{Technical Setup}

On a technical level, KIMA combines an unconventional screen interface-a holographic screen with real-time motion capture input, mathematic sound visualization, and quad surround sound. Abstracting holographic telepresence as a visualization interface, sound becomes the primary layer of presence. In two remote setups-one audio/visual, the other purely phoneticusers experience actions and interactions as a sense of one another's presence. The soundscape of the first space is transmitted as holographic imagery into the second space, where it allows for real-time modulation of both vision and sound. Through methods of reflection and refraction, holographic screens allow for projection into space as opposed to onto a surface. Users can then interact with imagery generated in real time-in this case, affecting both shape and sound.

This shared soundscape is visually represented as a cymatic pattern on the holographic screen. Actions and movements in this space modify visuals and the soundscape in what becomes a perpetual feedback loop of communication. Technically, KIMA distinguishes itself from other telepresence art installations in concentrating solely on the relationship of sound and matter through motion tracking on a holographic screen interface.

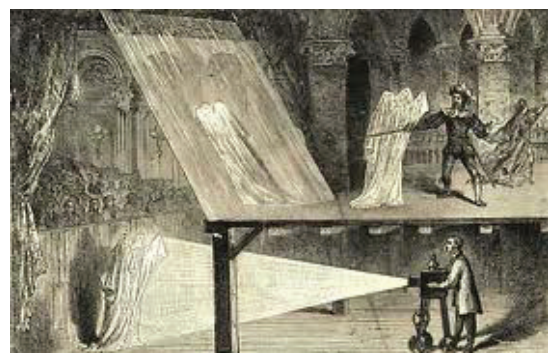

Figure 2. Edouard M. Charton, Classic Pepper's Ghost Display using glass, 1869.

Pepper's Ghost-based telepresence interfaces allow for life-sized, spatial representations of human interaction. Through the principles of reflection and refraction, images are projected into the physical space as opposed to onto a surface-allowing for onstage interaction with the visual form. This "holographic" representation (the perceived "hologram") appears as a life-sized image on stage to audience members in the auditorium. Overhead displays reference feedback streams for the presenter and the audience. As a spatial display technique, Pepper's Ghost projection serves as a platform for real-time communication (Figure 2).

In $K I M A$, this telepresence solution is adapted to display the mathematical interpretations of a sound stream rather than a life-sized person. The stream is then modified through motion capture (Microsoft Kinect) in real time for a two-way telepresence interaction. Real-timegenerated imagery is literally superimposed onto the performer and user. Real-time interaction between two spaces affects the shape of the structure, its spatial position, and the sound pattern it represents. This results in a perpetual interplay of communication.

In its first rendition, KIMA was presented at Kinetica Art Fair London 20I3, one of the UK's biggest forums for digital art—staged as a performance between a vocalist and a dancer. The presence of the vocalist is almost tangible. The result of the dancer's actions is relayed as interference pattern back to the vocalist. Whereas the sound from space A creates visuals in space $\mathrm{B}$, these visuals can be modified, translated, and redefined through the dancer's movementsconverging in an ever-changing joint telematic performance. Higher frequencies create different cymatic patterns, and the dynamic changes continuously through interaction. 


\section{Conceptual Setup}

As a telepresence performance, KIMA explores new strategies in offering both a performance and an interactive user installation to audiences. In the tradition of telepresence art (Roy Ascott, Eduardo Kac, et al.), the act of remote communication is the core subject matter of KIMA, yet its focus is firmly on the intrinsic relationship between audio and visual. How does sound generate matter, what happens when sound modifies matter (for example, when we as humans act as instruments and create music with our movement and presence across spaces)? These questions are investigated both on an academic and artistic level.

A number of artists have played with sound waves as either visible or invisible structures of communication. In 1970, Bernhard Leitner's Sound Cube used surround sound to immerse users in the experience of "geometric" sound, a walk-through audio architecture. David Bowen's tele-present water made use of water currents to deform a geometric structure at a remote location. Telepresence sound has been generated by Pauline Oliveros in her Deep Listening Institute since the I990s. Other than previous telepresence pieces, sound isn't the sole focus of attention, but more of a modus operandi for the larger theme of remote communication. Sound is understood as one of many strategies of remote communication between two spaces.

KIMA maps sound geometrically across two spaces, allowing the users to reference one another, to act as instruments within a composition they only partially influence. Performers or users thus form part of an ever-changing sound field that stands in the tradition of sound installation art (Wolf Vostell, Bill Fontana, et al.). KIMA "understands" sound patterns as part of a constantly changing force field that surrounds us, that we form part of - the essence of communication. $K I M A$ extrapolates this invisible environment by visualizing communication as real-time interactive interference patterns based on mathematical principles.

In ancient Greek, "kima" means "wave." In this telepresence environment, users experience the wave form in its sonic and physical forms as a traveling, continuously adapting stimulus—as an interface of interactions. In contemporary art production, cymatics-as visualization of phonetic waves - have been the inspiration of artists around the globe. Telepresence art's long tradition dates back to 1983, when Roy Ascott staged La Plissure du Texte, and was further pioneered in the early i990s (Paul Sermon [I], Tina Keane [2], Sommerer and Mignonneau [3] et al.). Recently, Paul Prudence-BioAcousticPhenomena (2010)—created visual references of cellular phenomena based on cymatic principles. $20 \mathrm{~Hz}$ by Ruth Jarman and Joe Gerhardt [4] is a sculpture generated by sound based on a geo-magnetic storm in the earth's atmosphere.

KIMA was conceived as a telepresence depicting cymatic patterns on a holographic interface. This visible structure of wave patterns is a continuum of user/performer interactions via body and voice in space. The work is a comment on the phenomenon of presence itself, at sound as an interference pattern, an orchestration of communication rather than mere representation of sound.

However, there is only limited available research on the intrinsic relationship between sound and visual telepresence. Academic research studies remain vague and inconclusive. With KIMA we attempt to fill this gap by providing a framework for future analysis of this specific aspect of telepresence. The work is distinguished from other telepresence installations in its specific focus on the intrinsic sound-matter relationship.

\section{Experiential Setup}

In $K I M A$, there are two types of communicative clues: On the one hand, users communicate with the space around them. They enter an invisible construct confined by the physical borders 
of the space-every movement induces a change in this sonic environment of echoes, frequencies, and geometrical attributes. Whereas one space allows for phonetic references only, the second space depicts a cymatic representation of these sound patterns, abstracting the presence of the other as interference patterns of communication.

The space itself and its exploration are central: Both performers are immersed in two distinctively prepared spaces, equipped with invisible sensors that enable navigation. A quad surround sound system provides feedback, and a holographic projection technology creates a volumetric sensation of light. Holographic screen displays use a transparent screen and methods of light reflection and refraction, thus allowing for the sensation of $3 \mathrm{D}$ without the need for $3 \mathrm{D}$ glasses. Employing visual cues and references that help to conjure the illusion of spatial depth perception, holographic screens map virtual information onto the real space-creating a plane of onstage interactivity between virtual images and live performers in a controlled-light environment. A dark environment along with a controlled-lighting solution plays a key role in this optical illusion.

By its very nature, a dark space engenders an immersive environment. How do we experience ourselves in such an environment once we sense another presence? On an experiential level, the dichotomy between you and me ceases to exist and gives way to an endless echo reverberating in both locations. In KIMA, information on the other presence is provided only through codecoded representation of sound or sound patterns transcoded into feedback loops. It is through this deformation of communication, through a veil of self and other, that we experience information on spatiality. KIMA looks at invisible presence on two levels: quad surround sound feedback loops and a visual representation of these sound patterns on the holographic screen. On this holographic screen, KIMA plays with notions of visibility and invisibility. Cymatic patterns only become visible when sound is emitted, i.e. when communication takes place.

"Invisibility" has been explored in contemporary art production and display. The Hayward Gallery's Invisible: Art about the Unseen, 1957-2012 was entirely dedicated to that theme. Jeppe Hein's Invisible Labyrinth (Centre Pompidou, 2005) explored architectonic presence through movement and sound relays [5]. In KIMA, the users remain almost invisible, as sound waves act as a medium of telepresence.

Whereas one space allows for sound as the sole reference to the other's presence, the other space displays a visual structure-a cymatic sound sculpture as representation of sound. Here, sound serves more as a prompter for cymatic effects - an interface for further interaction. This representation of sound as particle waveforms in a holographic environment is based on the voice of the other in the second space. Exploring both spaces, users can physically experience the difference between sonic and visual presence.

Holographic screen interfaces (here, a Musion Eyeliner screen) allow for spatial perception of visual information and for live onstage interaction with these visuals (in $K I M A$, via Microsoft Kinect). The Kinect camera traces z-depth information along with information on position, which serves as feedback to both Processing and Max/MSP scripts. Based on the principle of Pepper's Ghost, this creates an interface that can be experienced spatially. Any movement in this space creates further feedback both to the visual form on the display and the phonetic environment in both spaces. This cymatic telepresence setup can be performed (by professional performers) or simply explored. Both phenomena-cymatics and telepresence- have a long tradition in the visual arts, and both concepts have changed and evolved dramatically over time. 
The second communicative layer is the phonetic interaction with the other. Within the dark space, we almost expect to feel the other, yet we can only hear him or her exploring another space-wherever that might be. Location references of the other are generated through sound panning and mapping to the user's own environment. Location cues are augmented through echoes, reverberation, and frequency changes, and the fact that all we hear, feel, or see are interference patterns of sound-coincidences of communication.

Following Paul Wazclawick's studies in communication [6], not to communicate is virtually impossible. In $K I M A$, users are constantly exposed to a stream of information generated by their environments and users that explore them. Immersed in the KIMA setup, users transcend the boundaries of the physical space to communicate with similar spaces through different patterns of communication. KIMA explores a new route in telepresence art by creating an interface for a new form of presence that concentrates entirely on sound and matter as the nucleus of communication.

Processing code, whether displayed as cymatic pattern or purely audible, is based on the physical principles of cymatics-wave patterns that result from sonic frequency changes. Mathematically correct formulas build the framework for visual references that emerge in real time as representations of the invisible. KIMA as synaesthetic experience remains within the conceptual framework of telepresence art, while focusing on the sound-matter relationship through cymatics.

\section{Conceptual Issues: Cymatics and Telepresence}

KIMA has three main conceptual frameworks: telepresence as a concept and art form, cymatics as a research subject and art practice alike, and, in a wider context, synaesthetic art.

\section{Telepresence}

As a concept, telepresence dates back to the 1980s when the term was coined by Marvin Minsky in his seminal article "On Telepresence." Later conceptions focused on a mechanical telematics angle, aspects of virtual reality, and only in recent years on day-to-day communication. Minsky's techno-centric definition of the term gave way to proponents of a senso-motoric approach such as Thomas Sheridan's and ultimately focused on remote user experiences, such as the perceptual definition championed by the ISPR. The concept of telepresence relies heavily on the concept of remote presence-a user-centric idea of perceived non-mediation.

In art practice, pioneering experiments such as Paul Sermon's Think about the people now, which won the Golden Nica in 1991, were echoed by installations by Tina Keane, Julian Freud, and others through the 1990s and 2000s. Telepresence art has evolved dramatically from its early incarnation (La Plissure du Texte) through installations such as Ken Goldberg's Telegarden in the mid-r99os and Eduardo Kac's Ornitorrinco on the Moon in 1993.

The more the internet started to dominate everyday life, the more telepresence art became popular. Raphael Lozano Hemmer's telepresence installation for Ars Electronica, Displaced Emperors (1997), shifted the focus from people to architectural telepresence environments. Sommerer and Mignonneau's The Interactive Plant Growing (1993) had a botanical, organic context.

Over the last two decades, telepresence has found new channels in performance art, theater, and sculpture. In performance art, groups like Ghislaine Boddington's Body $>$ Data $>$ Space have experimented with new forms of telematic communication. In theater, the English National Theatre recently found a new outlet in telepresence performances by projecting plays live into 
cinemas across the UK. Telepresence sculptures represent a diverse spectrum, ranging from architectural art to classic sculptural forms. The architecture collective Assocreation connected two remote locations through a shared pneumatic floor interface with Bump in 2010 [7]. Michael Takeo Magruder's Data Flower (20I0) [8] presents a holographic sculpture as telepresence art- the visual form of an ever-changing flower based on a perpetual live internet stream of news feeds.

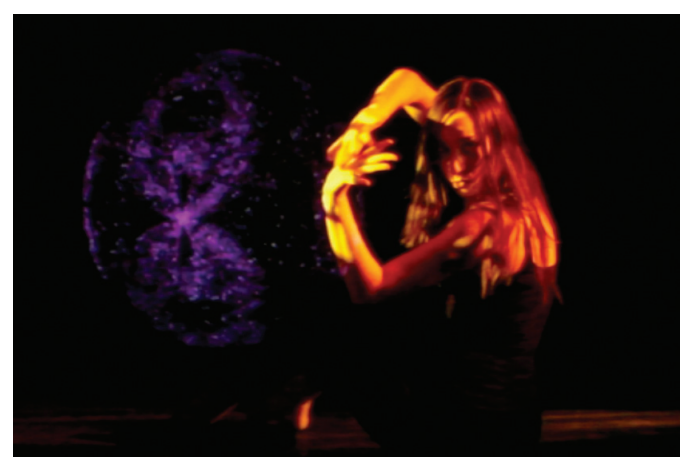

Figure 3. KIMA research performed at Kinetica London. ๑) 2013 Eugenia Emets.

KIMA is a telepresence environment, a performative space, a sound installation, and a holographic sculpture-a communication interface that allows users and performers to experience the spatial qualities of the physical space as a communicative infrastructure. KIMA's approach is new in that it shifts the focus from telepresence as remote representation to telepresence as audio/visual communication (Figure 3, 4).

\section{Cymatics}

The collective behind KIMA intends to use cymatic wave patterns in sound and image as a mediator for remote interactions. Cymatics, a terminology that derives from the Greek word $\kappa \tilde{u} \mu \alpha$ (wave), is known as the science of visible sound waves - the modal lines of particles or liquids within rigid environments as the result of sonic vibration. Ernst Chladni (1756-1827), the founder of acoustics, is known as the first to research vibrations and their effects on sand on rigid surfaces. Chladni demonstrated his famed laboratory setup at the French court and catalogued his studies to a certain degree.

In the 1960s, these experiments were further developed and academically contextualized by the Swiss scientist Hans Jenny, who looked at regularities in visual patterns and established a formula linking frequencies to visual patterns directly. His research and publications form a catalogue or atlas of wave patterns that now presents the backbone for future research for academics, musicians, and artists.

György Kepes, founder of the Center for Advanced Visual Studies at the Massachusetts Institute of Technology; Derek Kverno and Jim Nolen at Davidson College; and Thomas Cooper at Temple University all published diverse research papers on the subject. Recently Lewis Sykes from the Manchester Institute for Research in Art and Design investigated the subject in artistic and theoretic practice [9].

On a mathematical level, cymatic patterns have been discussed by Stewart and Colwell (I939) [Io], by Elmore and Head (I985) [II] and maybe most importantly by Paul Bourke, research associate professor at the University of Western Australia [I2].

In layman's terms, Chladni's patterns look at the movement of sound over rigid surfaces, at the acceleration of sine waves in a constrained environment such as a rectangular or round plate. Vibration patterns depend largely on the frequency itself as well as the rigidity of the surface and its confining boundaries. Modal residues settle where the speed of these frequencies equals zero. These points of zero acceleration are generally ordered symmetric "lines" arranged toward the center. Researchers have applied this model to three-dimensional space. 
Cymatics have influenced artists and researchers alike. Looking at cymatic patterns on a plate or in a volumetric form means to look at the intrinsic relationship between sound and sight. A Chladni plate ultimately acts as a medium for two dimensions to interact, as a mirror of their relationship. For KIMA, this contextual framework has been informative on mathematical, phonetic, artistic, and academic levels.

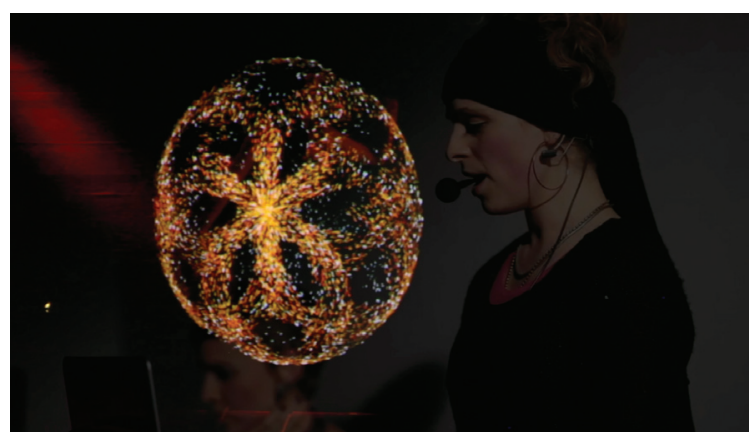

Figure 4. KIMA research performed at Kinetica London. $\odot 2013$ Eugenia Emets.

\section{Synaesthetic Art}

Audio/visual art has a long-standing tradition in cymatics: On a phonetic and symphonic level, R. Pellegrino's studies on “The Electronic Arts of Sound and Light” (1983) [13] and Alvin Lucier's composition "Queen of the South" stand out as artistic interpretations of the subject. Lewis Skyes' "Augmented Tonoscope" is a synaesthetic art project that concentrates on analog soundmatter visualizations [I4]. In the arts, Carsten Nicolai's Milch (2000) and Graham Wakefield's Chladni $2 \mathrm{D}$ and $3 \mathrm{D}$ Max/Msp patches have touched on the field of cymatics.

Experiments in synaesthesia, the relationship between sight and sound, go far beyond the arts and have been conducted in physics by Newton and in music by composers such as Louis Bertrand Castell over the centuries. In video and installation art, there is a long-standing tradition of experiments in synaesthesia spear-headed by Oskar Fischinger, Norman McLaren, John Whitney Sr. and Jr., and Mary Hallock-Greenwalt, whose color organs "Sarabet" and "Nourathar" were able to create sound while displaying correlating images at the same time.

As part of this long tradition of audio/visual art, KIMA is an attempt to be more than an audio/ visual instrument for the body. It is an instrument for two, a perpetual composition of synaesthesia, in which users become composers and choreographers and their interactions become visual symphonies.

On an academic level, KIMA looks at how sound enhances telepresence experiences for individual users. Research on surround sound in telepresence setups to date is still relatively inconsistent (compare: Lombard \& Ditton, 1997) [14, I5]. Studies diverge on the question of whether immersive sound enhances telepresence effectively and, if so, how much. KIMA investigates this intrinsic relationship. To this end, the physical setup will build the basis for future research, to be followed up by an academic evaluation based on interviews and questionnaires. KIMA is conceptualized as an investigation in audio/visual interrelations, an investigation of the role of sound in telepresence and the relationship between the visual and the phonetic.

\section{KIMA Technical Setup}

KIMA has three main dimensions:
A. Interactive immersive sound installation
B. A motion control interface
C. A visual interface 
We will discuss the installation in all three aspects:

A. KIMA as an immersive sound environment.

In $K I M A$, sound acts as the main communication interface between two spaces. On a technical level, the sound engine written in Max/MSP [16] and controlled via the Open Sound Control (OSC) protocol is the mediating application of the system. The sound engine also processes audio information and sends it via OSC [r7] to control the visuals. This process creates feedback loops for both environments. The audio signals are routed as raw audio data via Jacktrip and the Jackaudio Server

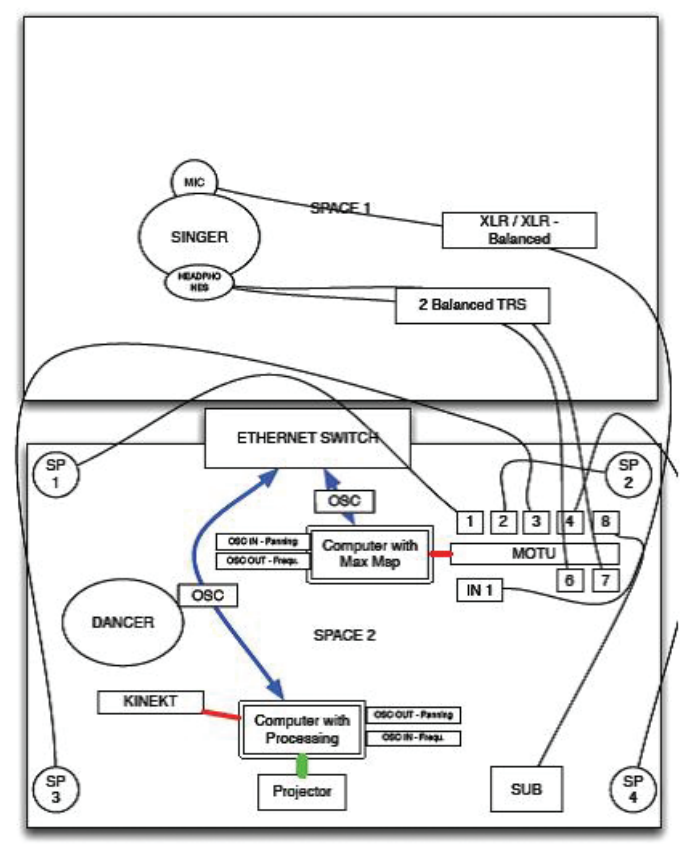

Figure 5. KIMA Technical Setup. @ 2013 Alain Renaud. (Cáceres et al., 2009) [I8] over the network. Interpretation of this audio stream in Processing [I9] is based on mathematical formulae of cymatics and sine wave functions of cymatics:

$\mathrm{u}(\mathrm{x}, \mathrm{y}, \mathrm{t})=\mathrm{A}^{*} \sin \left(\mathrm{Kx}^{*} \mathrm{x}\right) * \sin \left(\mathrm{Ky}^{*} \mathrm{y}\right) * \cos \left(\mathrm{w}^{*} \mathrm{t}\right)$

In environment $\mathrm{A}$, the audio is transmitted through contact microphones, capturing the low rumbling vibrations of the users in their space. A second condenser microphone communicates the conversations and noises emitted purposefully by the users. This sound signal is processed in real time over the network through Max/MSP and OSC into environment $\mathrm{B}$.

All sound sources of environments A and B are automatically mixed and streamed through an array of four speakers plus a subwoofer in environment A. At the same time, a flattened version in a two-channel mix is sent to environment $B$. In environment $B$, one contact microphone and one condenser microphone are installed to record ambience as well as user audio input for real-time transmission. Two individual streams are sent to a Max/MSP patch for the mixdown in the application. Users hear interference patterns in the two spaces, to which they are, of course, invited to respond in real time.

Whereas environment $A$ is set up as an immersive sound environment, environment $\mathrm{B}$ is equipped with a holographic projection system (Musion Eyeliner technology) to display cymatic wave patterns created in Processing along with stereo mixdown. Interference patterns are displayed both phonetically and visually as a materialization of communication. Sonic interactions create a sense of presence and dislocation, which results in an entirely new perception of environment, other, and self.

The overall sound environment represents an extension of naturally occurring, ambient sound signals produced by the users, thereby reinforcing the feeling of immersion and the level of engagements for the users (Figure 5). 
B. KIMA as a motion capture interface:

Motion information is used in environment $B$ to record and capture the position of the user and map it to the panning of the sound environment. The user in environment $A$ is therefore able to hear the sound in quad. Spatial information is transmitted in real time for the user in space A to interact with. Visuals in space B integrate motion information in the display script. Ergo space B acts as a visual playground, whereas space A consists of an immersive phonetic sculpture.

C. KIMA as a visual interface:

KIMA's visual display is programmed on the physical principles of cymatics: wave patterns created by different frequencies on rigid surfaces or inside a specified volume. The visual representation of interference patterns is based on mathematical formulae derived directly from the studies of wave patterns in contained spaces such as the Chladni plate. In line with

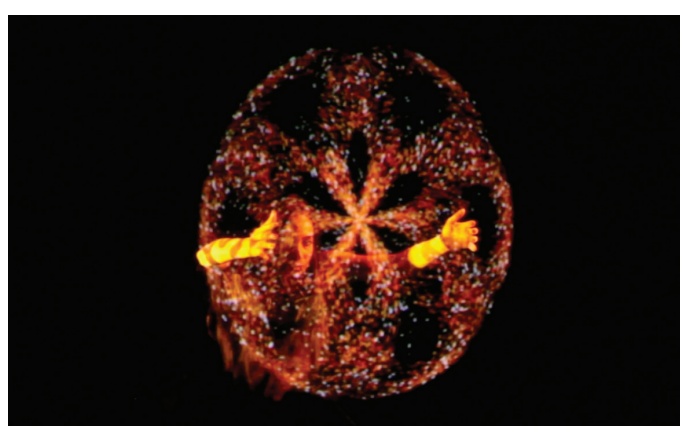

Figure 6. Real-time particles generated in Processing. ( $\odot 2013$ Eugenia Emets. existing studies on generative art, $K I M A$ 's visual interface follows a set of rules to make the visual representation easier to read and more intuitive to interact with. KIMA is based on mathematical formulae that derive directly from Hans Jenny's cymatics.

Various studies in both generative art and complexity theory state that highly ordered systems reduce visual entropy [20]. KIMA's telepresence experience is based on a highly ordered system to reduce entropy. The following rules have been laid out by the KIMA collective:

I. KIMA is autonomous: The aim is to create a completely self-generative, autonomous, and self-relying structure (Figure 6).

2. KIMA is cymatic: Both interfaces are based on principles of cymatics (sound creates and alters visual form).

3. $K I M A$ is communicative: Interference patterns of communication are its visual and phonetic representation.

4. KIMA is more than the sum of its parts: It consists of wave forms and particles that form together in entropy (Figure 7).

5. KIMA is audio/visual: The color spectrum is related to frequencies; sound panning represents locations.

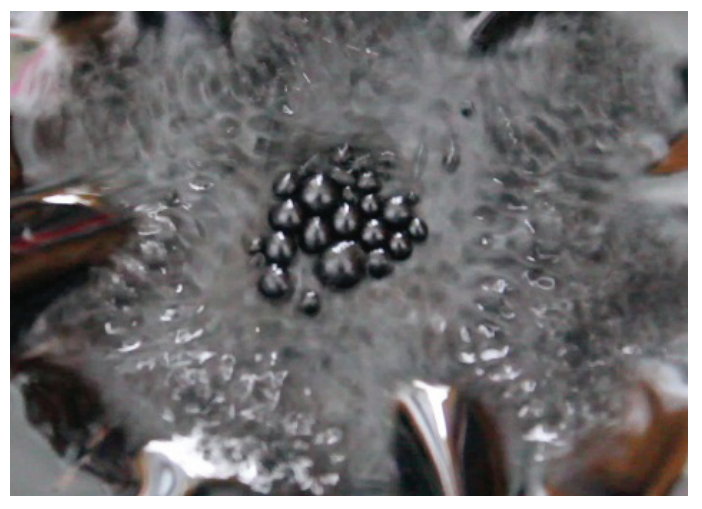

Figure 7. Before and After: Cymatic experiments using ferrofluids and the frequency range 432khz. (๑ 2013 Oliver Gingrich. 
6. KIMA is immersive: Physical space is mapped to a phonetic space, and different effects are attributed to it.

7. KIMA is user-focused: Visually and phonetically, centers are mapped to XYZcoordinates of users.

8. KIMA is generative: Audio loops entail repetition of the form.

9. KIMA is interactive: Once you stop interacting, the structure disappears.

Io. KIMA is communicative: The louder the signal, the more visible the structure becomes.

II. KIMA is responsive: The faster the user's movement, the faster the visual structure moves.

I2. KIMA is iconographic: Structure is created around the center.

13. KIMA is predictable: Amplitude of sound waves controls particle emission.

These guidelines illustrate the conceptual underpinnings of the KIMA code. KIMA is at once self-reliant and autonomous. Like any other generative art installation, it is based on physical principles and is rule-based. Interference patterns, the single economic denominator of user communication, are displayed as a visual representation. Users are turned into thereminsmusical instruments of a composition they can only influence but never solemnly direct. In this sense, $K I M A$ is organised under Paul Watzlawick's premise of the impossibility of noncommunication [2I]. KIMA makes use of a holographic interface (the Musion screen), which in itself is based on Pepper's Ghost projection methods.

\section{KIMA - Conclusions}

KIMA has a dual agenda, being at once academic research and an art installation, as well as being a phonetic immersive environment and a visual sculpture. KIMA is a telepresence experiment that plays with new technology: a holographic display technique based on the principle of Pepper's Ghost and a coded, programmed sound environment.

On an academic level, KIMA is at the basis of a research evaluation of the impact of immersive sound on telepresence. On an artistic level, it creates a performative space for the public to engage in. The research collective understands telepresence not as a communication technology (such as Skype or similar), but as a concept of presence in a remote location. KIMA is conceptualized as an immersive perceptive experience of communication. Stripped of a visual representation of the other, we experience communication as something that is surrounding our senses, omnipresent like the wave forms that cymatics generate.

As a non-verbal, non-facial communication process across distance, $K I M A$ invites the public to act as artist and create a phonetic environment that solemnly consists of communication.

KIMA also questions the conceptual context it operates in. Generative art is understood as art that consists of an autonomous system based on rules that form intelligent design. In the case of $K I M A$, a plurality of rules forms the structure of communication. On a visual level, these rules are based on cymatic patterns. On a phonetic level, the rules are interference patterns. This system is autonomous, yet dependent on user interference, on human interactions. Generative art aims 
for an autopoietic system that-once created - acts independently of human interference. KIMA is a generative art project, yet it requires human interactionsinterference patterns of communication-to come to life. In this sense, KIMA is both interactive art and a generative art project facilitated through telepresence. Generative art and interactive art are neighborly disciplines, yet clearly defined and demarcated across academic literature. KIMA tries to break with the conventions of this artificial dichotomy of terminologies in constituting a piece that can be attributed to

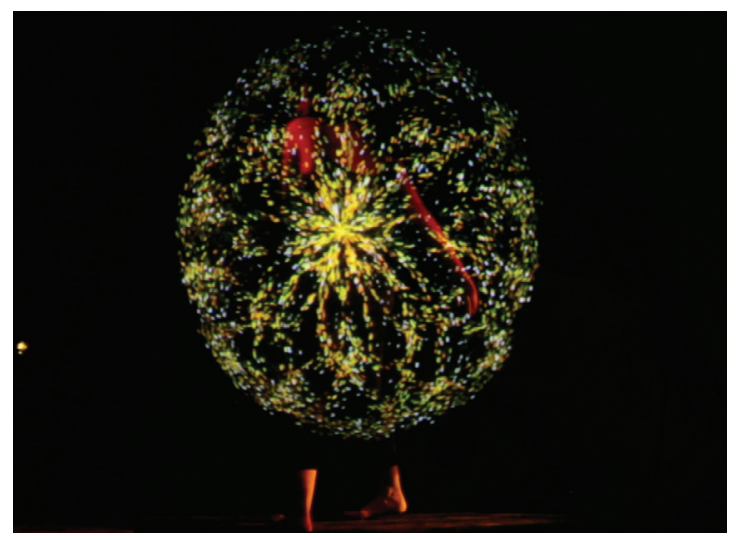

Figure 8. KIMA research, performed at Kinetica London. () 2013 Eugenia Emets. both worlds (Figure 8).

First and foremost, KIMA is a piece on communication and the impossibility of noncommunication. It understands spaces as representative immersive environments, as visual and phonetic echoes generated and created through communication. It is also an exploration of telepresence, of the essence of sound in establishing a sense of presence, a sense of other. This mediation process is the subject matter of the installation. Standing in a long tradition of pioneers (Roy Ascott, Paul Sermon) of telepresence art, we look at spatial environments as being filled with waveforms of communication. KIMA extrapolates these waves both on a visual and phonetic level, and reduces communication to an act of engagement.

In the next stages of project development, the research collective will focus on deeper exploration of cymatic aspects through research and realization of $3 \mathrm{D}$ variations of cymatic phenomena. Using the current model as a starting point, the aim is to create a more intuitive, flexible, and in a way more complex visual language for sound visualization, which would allow an even more immersive and fluid interaction experience. This development will proceed alongside experimentation both in the performance and installation setting with public participation. The collective plans to work with both voice and other instruments as sonic input for two-way communication. This will allow the project potentially to be used in the context of multiinstrument music performance in interaction with one or several dancers.

\section{References}

I. Sermon, Paul, Telematic Dreaming, 1992.

2. Keane, Tina, Couch, 1998.

3. Sommerer, Christa and Laurent Mignonneau, Interactive Plant Growing, 1992-1997.

4. Jarman, Ruth and Joe Gerhardt, $20 \mathrm{~Hz}$, accessed at $<$ semiconductorfilms.com $/ \mathrm{root} / 2 \mathrm{OHz} / 2 \mathrm{OHz}$.htm $>$ on II October, 2012.

5. Hein, Jeppe, Invisible Labyrinth, accessed at <jeppehein.net/pages/project_id.php?path=works\&id=I25> on II August, 2012.

6. Watzlawick, Paul et al., Pragmatics of Human Communication: A Study of Interactional Patterns, Pathologies and Paradoxes (New York: Norton, 1969).

7. Assocreation, Bump, accessed at <www.assocreation.com/project/bump/>.

8. Magruder, Michael Takeo, Data Flower (20IO), accessed at <www.takeo.org/nspace/nso34/>.

9. Sykes, Lewis, The Augmented Tonoscope, Manchester Institute for Research and Innovation in Art and Design, 20II. 
Io. Stuart, J.K., and R.C. Colwell, "The Calculation of Chladni Patterns," Journal of the Acoustical Society of America, Vol. II, No. I, I47-I5I (1939).

II. Elmore, William C. and Mark A. Heald, Physics of Waves (New York: Dover Publications, 1985).

I2. <paulbourke.net>.

I3. Pellegrino, Ronald, The Electronic Arts of Sound and Light (New York: Van Nostrand Reinhold, I983).

I4. Lombard, Matthew and Theresa Ditton, "At the Heart of It All: The Concept of Presence," Journal of Computer-Mediated Communication, Vol. 3, No. 2 (1997).

15. <phd.lewissykes.info>.

I6. Cycling '74, <www.cycling74.com>, accessed on II September, 2012.

17. Open Sound Control, <www.opensoundcontrol.org>.

I8. Cáceres, Juan-Pablo and Chris Chafe, "JackTrip: Under the Hood of an Engine for Network Audio," International Computer Music Conference Proceedings (ICMC 2009) (Ann Arbor, MI: Mpublishing, 2009).

19. Reas, Casey et al., Processing: A Programming Handbook for Visual Designers and Artists (Cambridge, MA: MIT Press, 2007).

20. Lloyd, Seth, Programming the Universe: A Quantum Computer Scientist Takes on the Cosmos (New York: Vintage, 2006).

2I. Watzlawick, Paul et al., Pragmatics of Human Communication: A Study of Interactional Patterns, Pathologies and Paradoxes (New York: Norton, 1969).

\section{Bibliography}

Ascott, Roy, La Plissure du Texte (1983).

Leitner, Bernhard, Sound Cube, accessed at <www.bernhardleitner.com/works>, II September, 2012.

Boden, Margaret and Ernest Edmonds, "What Is Generative Art?" Digital Creativity, Vol. 20, No. I-2, 2I-46 (20IO).

Bornstein, Marc, review of Symmetry in Science and Art by A.V. Shubnikov and V.A. Kopstik, Leonardo, Vol. Io, No. 4, 334 (1977).

Bowen, David, Tele-Present Water, accessed at <www.creativeapplications.net/maxmsp/tele-present-watermaxmsp-arduino/> on II September, 20I2. Dechevsky, Lubomir and Joakim Gundersen, "On the Scientific Visualization of Complex-Valued Functions of One Complex Variable," Applications of Mathematics in Engineering and Economics: 35th International Conference: AMEE 2009 (AIP Conference Proceedings), ed. George Venkov et al. (Melville, NY: American Institute of Physics, 2009).

Goldberg, Ken, Telegarden, 1995 .

Heeter, Carrie, “Being There: The Subjective Experience of Presence,” Presence, Vol. I, No. 2, 262-27I (I992).

JackAudio, <jackaudio.org>, accessed on II September, 2012.

Jenny, Hans, Cymatics: A Study of Wave Phenomena and Vibration, Vol. I, 1967 (Newmarket, NH: MACROMedia Publishing, 200I).

Kacs, Eduardo, Ornitorrinco on the Moon, 1993.

Kramer, Gregory, "Sound and Communication in Virtual Reality," Communication in the Age of Virtual Reality, ed. Frank Biocca and Mark R. Levy (Hillsdale, NJ: Lawrence Erlbaum, 1995) 259-276.

Lozano, Hemmer Raphael, Displaced Emperors, Ars Electronica Linz, 1997.

Pellegrino, Ronald, The Electronic Arts of Sound and Light (New York: Van Nostrand Reinhold, 1983).

Sheridan, Thomas B., "Musings on Telepresence and Virtual Presence," Presence, Vol. I, No. I, I20-I26 (1992).

Short, John et al., The Social Psychology of Telecommunications (London: Wiley, 1976).

Wright, Matthew and Adrian Freed, "Open Sound Control: A New Protocol for Communicating with Sound Synthesizers," International Computer Music Conference Proceedings (ICMC 1997) (Ann Arbor, MI: Mpublishing, I997). 